\title{
Detection of body postures and movements in ambulatory adults with cerebral palsy: a novel and valid measure of physical behaviour
}

Everett A. Claridge 1,2,3, Rita J. G. van den Berg-Emons ${ }^{3}$, Herwin L. D. Horemans ${ }^{3}$, Wilma M. A. van der Slot ${ }^{3,4}$, Nick van der Stam ${ }^{3}$, Ada Tang ${ }^{1}$, Brian W. Timmons ${ }^{2,5}$, Jan Willem Gorter ${ }^{1,2}$ and Johannes B. J. Bussmann ${ }^{3^{*}}$

\begin{abstract}
Background: Accurate measurement of physical behaviour is paramount to better understand lifestyle, health, and functioning, particularly in adults with physical disability as they may be at higher risk of sedentary lifestyle and subsequent negative health consequences. This study aimed: 1) to evaluate the criterion validity of a novel and clinically applicable activity monitor (AM, Activ8), in the detection of body postures and movements in adults with spastic cerebral palsy (CP); and 2) to evaluate the extent that the AM's positioning affects validity.

Methods: In this cross-sectional study, 14 ambulatory adults with CP [9 men; mean (SD) age, 35.4 (13.1) years] performed standardized activities while wearing three Activ8 monitors - frontolateral thigh (primary position), frontal thigh, and pant pocket - and being video recorded (criterion measure). AM activity output was compared to synchronized video recordings. Absolute (seconds) and relative [(video time-AM time)/mean time, \%] time differences between methods were calculated. Relative time differences of $<10 \%$ were indicative of good validity. Comparison of AM attachment positions was completed using Spearman Rho correlation coefficients and Meng's tests.

Results: Criterion validity of the AM (frontolateral thigh) was good (average relative time differences: $0.25 \%$ for sitting, 4.69\% for standing, 2.46\% for walking, 1.96\% for upright activity, 3.19\% for cycling), except for running (34.6\%). Spearman Rho correlation coefficients were greater between video/frontolateral thigh position than video/ frontal thigh position and video/pant pocket position for body posture and movement categories sitting, standing, walking, and upright activity ( $p<0.01$ for all).

Conclusions: The AM, positioned on the frontolateral thigh, demonstrated good criterion validity in ambulatory adults with CP. Though the Activ8 offers potential as an objective measure of physical activity, appropriate positioning is paramount for valid measurement.
\end{abstract}

Keywords: Cerebral palsy, Activity monitor, Accelerometry, Physical activity, Physical behaviour, Objective measurement

\footnotetext{
* Correspondence: j.b.j.bussmann@erasmusmc.nl

${ }^{3}$ Department of Rehabilitation Medicine, Erasmus MC University Medical

Centre, P.O. Box 2040, 3000 Rotterdam, CA, Netherlands

Full list of author information is available at the end of the article
}

(c) The Author(s). 2019 Open Access This article is distributed under the terms of the Creative Commons Attribution 4.0 International License (http://creativecommons.org/licenses/by/4.0/), which permits unrestricted use, distribution, and reproduction in any medium, provided you give appropriate credit to the original author(s) and the source, provide a link to the Creative Commons license, and indicate if changes were made. The Creative Commons Public Domain Dedication waiver (http://creativecommons.org/publicdomain/zero/1.0/) applies to the data made available in this article, unless otherwise stated. 


\section{Introduction}

Physical behaviour, which encompasses both physical activity (PA) and sedentary behaviour (SB), plays an important role in the health and functioning of adults [1-4]. Decreased PA and increased SB pose risks for negative health outcomes in adults, notably increased risk for cardiovascular disease and early mortality $[5,6]$. To mitigate the possible health consequences of inactivity, clinicians are encouraged to promote PA to patients as a preventive health measure [7-9]. Promotion of physical behaviour is particularly important for adults with cerebral palsy $(\mathrm{CP})$, as they have low levels of PA, increased SB, and reduced cardiorespiratory fitness [10-14]. Furthermore, as people with $\mathrm{CP}$ age, they undergo a decline in functioning $[15,16]$ that can further reduce PA and place them at risk of developing co-morbidities [17].

Knowledge of physical behaviour is essential for clinicians to effectively promote PA and limit SB in clinical populations $[1,9,18]$. Compared to self-report measures of physical behaviour, objective measures such as activity monitors (AMs) are generally considered more robust and sensitive to change [19]. Often used to assess physical behaviour over extended periods of time, AMs play an important role when evaluating interventions aiming to change physical behaviour [20,21]. AMs, which include accelerometers, are objective in nature and can provide feasible measurement of $\mathrm{PA}$ in a free-living setting.

The recently developed Activ8 Physical Activity Monitor $^{\mathrm{a}}$ (Remedy Distribution Ltd., Valkenswaard, The Netherlands) offers a novel, objective method to measure PA. The main difference with other consumer monitors is that it provides information on distinct body postures and movements (P\&Ms), whereas other AMs, such as the ActiGraph, mainly provide estimates of energy expenditure or evaluate time within PA intensities [22]. Additionally, the Activ8 is unobtrusive $(34 \times 30 \times$ $10 \mathrm{~mm}, 20 \mathrm{~g}$ ) and cost-friendly (€99), offering potential use in rehabilitation research and clinical settings. Activ8 users have the ability to view their recorded time spent in PA and SB on a personal computer or smart phone through the visually appealing dashboard (https://www. activ8all.com/app-dashboard/). Researchers and clinicians can add a coaching account, providing insight into their patients' physical behaviour. Furthermore, features such as goal setting and an integrated feedback system may compel users to maintain a healthy and active lifestyle.

Preliminary evidence for criterion validity of the Activ8 AM exists in healthy adults (unpublished observations) and adults after stroke [23]. However, for individuals with atypical gait or movement patterns, like those with spastic $\mathrm{CP}$, the validity of the device to detect body P\&Ms needs to be determined before extending its use in larger research studies or in the clinical setting. Furthermore, though Activ8 was designed and calibrated for pocket placement, aforementioned preliminary evidence for validity of the Activ8 AM suggests that direct attachment to the thigh would be more advantageous due to issues regarding varying pocket positions, sizes and the potential for excessive movement. Therefore, the primary objective of this study was to evaluate the criterion validity of the Activ8 AM in the measurement of body $\mathrm{P} \& \mathrm{Ms}$ in ambulatory adults with spastic CP, the most common type of CP [24], compared to direct video observation (criterion measure). The secondary objective was to determine the extent that positioning of the Activ8 accelerometer on the thigh or in the pocket affected the validity of the device.

\section{Methods}

\section{Participant Recruitment \& Eligibility}

A convenience clinical sample of fourteen adults with spastic CP [9 men; mean (SD) age, 35.4 (13.1) years] was recruited between February and June 2016. The sample size was based on comparable studies evaluating the validity of AMs with similar features, namely the VitaMove and the activPAL, in both children and adults with disability [25-27]. Participants were recruited through Rijndam Rehabilitation in Rotterdam, the Netherlands, by invitation from physicians once eligibility was determined and interest to participate was expressed. In addition, participants of past research studies who had indicated they would be interested in future research studies were contacted by mail and eligibility was confirmed by telephone. We aimed to recruit an equal distribution of participants across Gross Motor Function Classification System (GMFCS) levels I, II, and III.

The inclusion criteria were: $\geq 18$ years of age; diagnosis of spastic CP; ambulatory, with or without the use of assistive devices; and physically able to perform the activities in the assessment protocol. Participants were excluded if they had: disabilities other than $\mathrm{CP}$ affecting daily PA; severe cognitive disorder; insufficient comprehension of either English or Dutch to follow instructions for testing, as determined during a screening telephone conversation; or orthopaedic surgery within the past 6 months. All participants provided written informed consent. The study was approved by the Medical Ethics Committee of Erasmus MC University Medical Centre, Rotterdam, NL.

\section{Measures}

\section{Activ8 activity monitor}

The Activ8 AM (see Fig. 1a) contains a tri-axial accelerometer (MMA 8541, Freescale Semiconductor, Denver, USA) sensitive to high accelerations, all in the human activity spectrum ( $\pm 4.0 \mathrm{~g}$ in magnitude). The Activ8 has 




Fig. 1 a. The Activ8 Physical Activity Monitor. b. Frontal (superior to patella) and frontolateral $(2 \mathrm{~cm}$ laterally from frontal) thigh positioning of Activ8

a sampling rate of $12.5 \mathrm{~Hz}$ and uses a 14-bit analog-todigital converter. The Activ8 monitor then stores digitized acceleration samples using a First In, First Out (FIFO) data buffer that can hold 32 samples; therefore, each FIFO buffer holds $2.56 \mathrm{~s}$ of activity data.

Automated analysis of the angular position of the Activ8 AM, based on the signal from the $\mathrm{z}$-axis accelerometer, as well as the vector magnitude acceleration (from $\mathrm{x}, \mathrm{y}$, and $\mathrm{z}$-axes) allows raw acceleration samples to be converted into body P\&M activity classes. These include the classes of non-wear/lying, sitting, standing, walking, bicycling, and running. The monitor was set to record body $\mathrm{P} \& \mathrm{M}$ data using a 5 -s epoch, the lowest epoch setting available, allowing researchers to determine the amount of time spent in each Activ8 class. The Activ8 is unable to distinguish transient lying $(<5 \mathrm{~min})$ from sitting, as the angular position of the monitor would remain the same for both static postures. Body P\&M activity class data were downloaded using the professional Activ8 software (Version 2.1.0.22) and were used for comparison with video observation data.

\section{Video observation}

A handheld digital video camera was used as the reference method for detecting body P\&Ms. Video recordings were watched two times, at half speed, by one researcher. These recordings were analysed and scored independently of the Activ8 data output. Using a 1-s time resolution, video recorded activity was assigned one of the following categories based on pre-defined definitions: non-wear; lying; sitting; standing; standing with movement; walking (including shuffling); stair climbing; running; bicycling; or transition (sit-to-stand/stand-to-sit).

The following steps were taken to minimize bias when scoring the criterion measure of video observation: 1) each 1-s video frame was coded based on rigid definitions of body P\&M categories; 2) the video recording of the first participant was analysed by both author EC and an external researcher experienced with use of the Active8 - any discrepancies or areas of contention were discussed and a precedent was set for future issues encountered; and 3) any new issues encountered or periods of ambiguous body P\&Ms during subsequent video recordings were highlighted and discussed between author $\mathrm{EC}$ and the external researcher until an agreed solution was reached.

\section{Monitor placement on the body}

Participants were asked to wear three Activ8 monitors, two taped directly on the thigh with Tegaderm $^{\text {in }}$ skin tape (frontolateral thigh and frontal thigh locations - see Fig. 1b) and one placed in the pocket of the trousers. The frontal thigh position was located 1/3rd the length of the thigh as measured from the greater trochanter to the patella. The frontolateral thigh location was located at the same height as the frontal thigh position but set 2 $\mathrm{cm}$ laterally from the thigh's midline. This frontolateral position was our primary position as AM placement here more closely resembles the pant pocket position, for which the Activ8 was originally calibrated. All three monitors were placed at the side least affected by spasticity, or simply at the right side if there was no difference in spasticity among lower limbs.

\section{Procedure}

All participants performed a series of activities according to a standardized protocol in a simulated free-living environment within the occupational therapy department and human movement laboratory at Erasmus MC, Rotterdam, NL. The Activity Protocol (see Table 1) consisted of 
Table 1 Activity Protocol

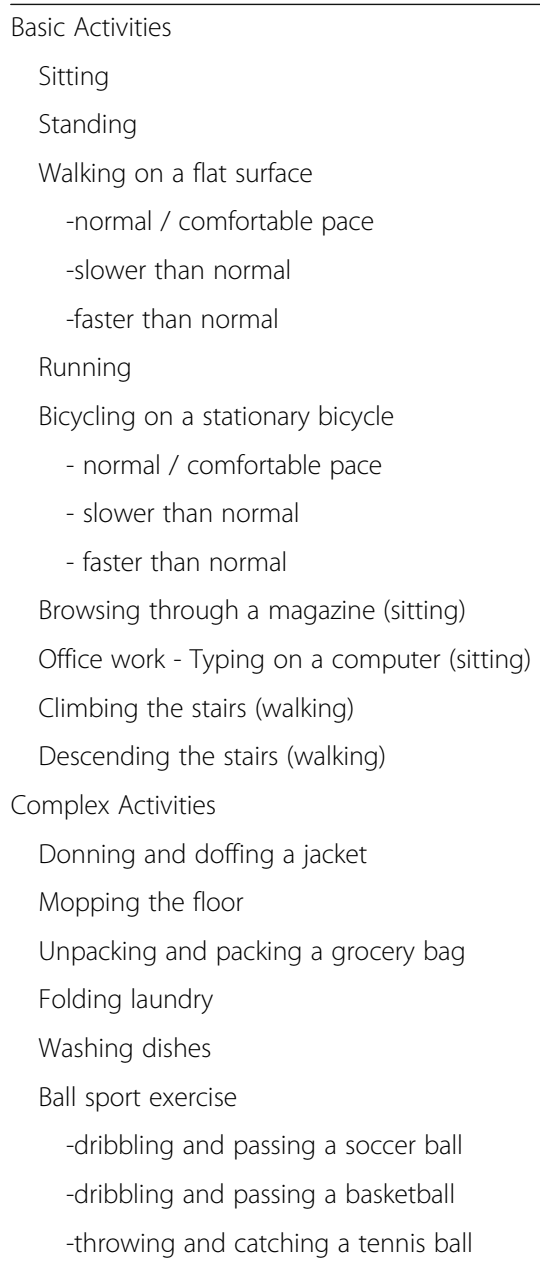

meaningful activities representative of daily life in individuals with CP, as informed by the literature [25, 28, 29]. This protocol included both basic activities, involving just one posture or movement (e.g. standing, walking), and complex activities, involving a number of P\&Ms (e.g. mopping the floor consists of standing and walking), and was completed in the same order each assessment. Participants were filmed for the duration of the assessment, while wearing the AMs. All activities were timed to be 80 $\mathrm{s}$ duration; less if the activity was completed before then (e.g. climbing two flights of stairs varied in duration). Start and stop times were noted during measurement, to ensure measures could be properly synchronized and compared. Body P\&M activity class data were downloaded using professional Activ8 software upon completion of the assessment.

Activ8 activity class data (5-s resolution) was then compared to the synchronized video observation (1-s resolution). Activ8 epochs do not display the order that numerous body P\&Ms were performed. For example, a 5 -s epoch may indicate that $2 \mathrm{~s}$ were spent standing and
$3 \mathrm{~s}$ were spent walking, but it does not specify whether standing preceded walking or vice-versa. Therefore, each performed activity from the Activity Protocol was analysed as a whole. The first and last epoch sample were removed due to the potential overlap of the Activ8 epoch with the start and end time of each activity. Additionally, to allow comparison between measures, the following video classes were merged: lying to sitting and stair climbing to walking. The video class 'standing with movement' denoted ambiguous movement that fell between standing and walking classes (e.g. lunging to reach under a desk while mopping; see Table 2).

\section{Data analysis}

Absolute time (|Activ8 time-video time|, seconds) and relative time [(|Activ8 time-video time $\mid) /$ video time, \%] differences were calculated for each $P \& M$ category for each Activ8 position, based on simple sum values of video and AM time. Additionally, median and quartile values of absolute and relative time differences were determined. For the primary analyses, an average relative difference of $10 \%$ or less between video and Activ8 measures was considered an acceptable level for criterion validity. This acceptable difference has been used in previous validation studies and is indicative of satisfactory validity [25, 30, 31].

Basic and complex activities (see Table 1) were analysed separately. "Standing with movement" time was allocated to the standing class during basic standing activities and to the walking class during basic walking activities. For complex activities, all standing, standing with movement, and walking time was allocated to a merged category labelled 'upright activity' (see Table 2).

To estimate agreement between measures on an individual level, Bland-Altman plots [32] were completed using data collected from the three Activ8 monitor

Table 2 Corresponding body P\&M categories between video and Activ8 measures

\begin{tabular}{|c|c|c|}
\hline \multirow[t]{2}{*}{ Video } & \multicolumn{2}{|l|}{ Activ8 } \\
\hline & $\begin{array}{l}\text { Basic activity } \\
\text { protocol }\end{array}$ & $\begin{array}{l}\text { Complex activity } \\
\text { protocol }\end{array}$ \\
\hline Non-wear & Non-wear & Non-wear \\
\hline Lying & Sitting & Sitting \\
\hline \multicolumn{3}{|l|}{ Sitting } \\
\hline Standing & Standing & Upright activity \\
\hline Standing with movement & Standing OR Walking* & \\
\hline Walking & Walking & \\
\hline \multicolumn{3}{|l|}{ Stair climbing } \\
\hline Running & Running & Running \\
\hline Bicycling & Bicycling & Bicycling \\
\hline
\end{tabular}

*dependent on the activity itself 
positions. Plots compared the difference between measures (Activ8 time-video time, in sec) to the criterion, reference measure (video time) as recommended by Krouwer [33].

To compare data from the three Activ8 positions, the statistical program Stata (Version 13.1, Texas, USA) was used. Using the total time allocated to each class, Spearman Rho correlation coefficients were calculated between measures. The following categories were used to interpret correlation coefficients: 0.0 to 0.4 (poor to low agreement), 0.4 to 0.6 (low to moderate agreement), 0.6 to 08 (moderate to strong agreement), and 0.8 to 1.0 (very strong agreement) [34]. Meng's tests [35] were then completed to determine whether correlation coefficients between video/frontolateral thigh Activ8, video/frontal thigh Activ8, and video/pant pocket Activ8 were statistically different.

\section{Results}

Participant characteristics are displayed in Table 3. Half of the participating adults had unilateral spastic CP (hemiplegic distribution, $n=7$ ) and half had bilateral spastic CP (diplegic or quadriplegic distribution, $n=7$ ). As a result of lower-limb spasticity, four participants had a prominent crouched stance or a crouch gait pattern (i.e. $>20$ degrees knee flexion). GMFCS levels were as follows: level I $(n=6)$, level II $(n=5)$, and level III $(n=$ 3). Missing data resulted from lack of Activ8 equipment for participant number 3 and trousers lacking pockets for participant number 14 .

The absolute and relative time differences between video and Activ8 measures for basic and complex activities are reported in Table 4. For the frontolateral thigh position, the average relative time differences between video and Activ8 methods were $<10 \%$ for detection of sitting $(0.25 \%)$, standing $(4.69 \%)$, walking $(2.46 \%)$, and bicycling (3.19\%), but $>10 \%$ for running (34.6\%). Average relative time differences range from $2.92 \%$ (bicycling) to $34.9 \%$ (running) for the frontal thigh position and $0.41 \%$ (sitting) to $35.4 \%$ (running) for the pant pocket position.

Bland-Altman plots display biases between measurement tools and between-subject variability for each detected P\&M (see Fig. 2). In these plots, the middle solid line represents the mean difference between methods and the wide dashed lines represent the upper and lower limits of agreement. The mean difference was smallest for the frontolateral thigh position $(0.03,95 \% \mathrm{CI}=-5.67$ to $5.62 \mathrm{~s})$ and larger for the frontal thigh $(0.05,95 \% \mathrm{CI}=$ - 13.2 to $13.1 \mathrm{~s})$ and pant pocket position $(0.07,95 \%$ $\mathrm{CI}=-13.4$ to 13.3$)$.

Spearman Rho correlation coefficients between video observation and Activ8 AMs were calculated. For the frontolateral thigh position, correlation coefficients between Activ8 and video measures were moderate to strong ranging from 0.49 to 0.99 . Correlation coefficients range from -0.04 to 0.86 for the frontal thigh position and 0.14 to 0.79 for the pant pocket position. Meng's tests (see Table 5) revealed that the correlation coefficients from video/frontolateral thigh Activ8 position were significantly larger than the correlation coefficients from video/front thigh Activ8 and video/pant pocket Activ8 for the P\&M categories of sitting, basic standing, basic walking, and upright activity ( $p<0.01$ for all). The

Table 3 Participant Characteristics

\begin{tabular}{|c|c|c|c|c|c|c|c|}
\hline \multicolumn{8}{|c|}{ Activ8 Monitors Worn } \\
\hline Participant No. & Age (years) & Gender & Frontal Thigh & Frontolateral Thigh & Pant Pocket & Limb Distribution & GMFCS-E\&R Level \\
\hline 1 & 23 & $F$ & $x$ & $x$ & $x$ & Hemiplegia & I \\
\hline 2 & 26 & M & $x$ & $x$ & $x$ & Hemiplegia & । \\
\hline 3 & 28 & F & $x$ & & $x$ & Diplegia & III \\
\hline 4 & 23 & M & $x$ & $x$ & $x$ & Hemiplegia & । \\
\hline 5 & 48 & M & $x$ & $x$ & $x$ & Diplegia & $\|$ \\
\hline 6 & 46 & M & $x$ & $x$ & $x$ & Quadriplegia & III \\
\hline 7 & 55 & M & $x$ & $x$ & $x$ & Hemiplegia & । \\
\hline 8 & 58 & M & $x$ & $x$ & $x$ & Quadriplegia & $\|$ \\
\hline 9 & 21 & $\mathrm{~F}$ & $x$ & $x$ & $x$ & Hemiplegia & । \\
\hline 10 & 43 & M & $x$ & $x$ & $x$ & Diplegia & $\|$ \\
\hline 11 & 24 & $\mathrm{~F}$ & $x$ & $x$ & $x$ & Diplegia & $\|$ \\
\hline 12 & 29 & M & $x$ & $x$ & $x$ & Hemiplegia & $\|$ \\
\hline 13 & 27 & M & $x$ & $x$ & $x$ & Hemiplegia & I \\
\hline 14 & 45 & $\mathrm{~F}$ & $x$ & $x$ & & Diplegia & III \\
\hline
\end{tabular}


Table 4 Total time, absolute time difference, and relative time difference between video observation and Activ8 AM

\begin{tabular}{|c|c|c|c|c|c|c|c|c|}
\hline \multirow[b]{2}{*}{ Frontolateral Thigh } & \multicolumn{2}{|l|}{ Video time (sec) } & \multicolumn{2}{|l|}{ Activ8 time (sec) } & \multicolumn{2}{|c|}{ Absolute Time Difference (sec) } & \multicolumn{2}{|c|}{ Relative Time Difference (\%) } \\
\hline & Median (Q1, Q3) & $\begin{array}{l}\text { Simple sum } \\
(n=13)\end{array}$ & Median (Q1, Q3) & $\begin{array}{l}\text { Simple sum } \\
(n=13)\end{array}$ & Median (Q1, Q3) & $\begin{array}{l}\text { Total }^{\mathrm{b}} \\
(n=13)\end{array}$ & Median (Q1, Q3) & Average $^{b}$ \\
\hline \multicolumn{9}{|l|}{ Basic Activities } \\
\hline Sitting & $237(232,256)$ & 3175 & $237(232,256)$ & 3183 & $0(0,0)$ & 8 & $0.00(0,0)$ & 0.25 \\
\hline Standing & & 1066 & $78(74,81)$ & 1016 & $3(2,7)$ & 50 & $4.00(2.4,8.0)$ & 4.69 \\
\hline Walking & $82(73,86)$ & 3706 & $286(261,311)$ & 3615 & $4(3,8)$ & 91 & $1.26(1.0,3.0)$ & 2.46 \\
\hline Bicycling & $291(268,302)$ & 2598 & $229(155,232)$ & 2515 & $0(0,7)$ & 83 & $0.43(0,10)$ & 3.19 \\
\hline Running & $\begin{array}{l}229(221,232) \\
69(0,72)\end{array}$ & 622 & $68(3,83)$ & 837 & $5(0,16)$ & 215 & $16.9(8.8,47)$ & 34.6 \\
\hline \multicolumn{9}{|l|}{ Complex Activities } \\
\hline Upright Activity ${ }^{a}$ & $460(348,544)$ & 6072 & $463(347,544)$ & 5953 & $1(0,9.5)$ & 119 & $0.30(0,2.7)$ & 1.96 \\
\hline Frontal Thigh & Median (Q1, Q3) & $\begin{array}{l}\text { Simple sum } \\
(n=14)\end{array}$ & Median (Q1, Q3) & $\begin{array}{l}\text { Simple sum } \\
(n=14)\end{array}$ & Median (Q1, Q3) & $\begin{array}{l}\text { Total } \\
(n=14)\end{array}$ & Median (Q1, Q3) & Average $^{b}$ \\
\hline \multicolumn{9}{|l|}{ Basic Activities } \\
\hline Sitting & $242(231,259)$ & 3474 & $245(233,300)$ & 3722 & $0(0,0.75)$ & 248 & $0.00(0,0.29)$ & 7.14 \\
\hline Standing & & 1139 & $75(71,78)$ & 861 & $6(1.5,16)$ & 278 & $7.30(1.9,21)$ & 24.4 \\
\hline Walking & $79(75,84)$ & 3877 & $279(250,302)$ & 3604 & $11(1.8,29)$ & 273 & $3.68(0.63,14)$ & 7.04 \\
\hline Bicycling & $290(278,299)$ & 2809 & $224(208,228)$ & 2891 & $0(0,5.3)$ & 82 & $0.00(0,2.7)$ & 2.92 \\
\hline Running & $\begin{array}{l}226(222,232) \\
63(0,74)\end{array}$ & 628 & $64(0,94)$ & 847 & $2.5(0,22)$ & 219 & $19.2(5.3,42)$ & 34.9 \\
\hline \multicolumn{9}{|l|}{ Complex Activities } \\
\hline Upright Activity ${ }^{a}$ & $498(332,568)$ & 6277 & $470(281,566)$ & 5795 & $5(0,19)$ & 482 & $1.30(0.39,3.34)$ & 7.68 \\
\hline Pant Pocket & Median (Q1, Q3) & $\begin{array}{l}\text { Simple sum } \\
(n=13)\end{array}$ & Median (Q1, Q3) & $\begin{array}{l}\text { Simple sum } \\
(n=13)\end{array}$ & Median (Q1, Q3) & $\begin{array}{l}\text { Total }^{\mathrm{b}} \\
(n=13)\end{array}$ & Median (Q1, Q3) & Average $^{b}$ \\
\hline \multicolumn{9}{|l|}{ Basic Activities } \\
\hline Sitting & $241(232,257)$ & 3207 & $236(223,261)$ & 3194 & $0(0,1)$ & 13 & $0.00(0,0.6)$ & 0.41 \\
\hline Standing & & 990 & $80(75,85)$ & 1015 & $7(1,10)$ & 25 & $8.33(1.3,57)$ & 2.53 \\
\hline Walking & $79(74,86)$ & 3532 & $278(244,330)$ & 3614 & $35(7,49)$ & 82 & $11.2(2.5,34)$ & 2.32 \\
\hline Bicycling & $288(252,307)$ & 2608 & $230(107,231)$ & 2292 & $1(0,69)$ & 316 & $5.75(0,44)$ & 12.1 \\
\hline Running & $\begin{array}{l}230(223,231) \\
71(0,75)\end{array}$ & 624 & $65(5,95)$ & 845 & $9(0,20)$ & 221 & $26.7(17,66)$ & 35.4 \\
\hline \multicolumn{9}{|l|}{ Complex Activities } \\
\hline Upright Activity $^{a}$ & $506(242,584)$ & 6023 & $482(223,559)$ & 5762 & $3(0,23)$ & 261 & $2.01(0.4,6.4)$ & 4.33 \\
\hline
\end{tabular}

a. Upright Activity; the sum of Standing, Standing with Movement, and Walking categories

b. Based on simple sum values

correlation coefficients from video/Activ8 measures were not different among AM wear locations for detection of bicycling or running.

\section{Discussion}

This study evaluated the criterion validity of the Activ8 AM in the detection of body P\&Ms during daily life activities in ambulatory adults with spastic
$\mathrm{CP}$. The low absolute and relative differences in time between video and Activ8 at the frontolateral thigh position suggest the Activ8 is an appropriate tool to detect body P\&Ms in ambulatory adults with $\mathrm{CP}$. One important caveat to this conclusion is the detection of running, which was poorly detected by the Activ8 ( $>10 \%$ average relative time difference). The detection of body P\&Ms from the frontal thigh and pant pocket positions was acceptable $(<10 \%$ difference $)$ for the 

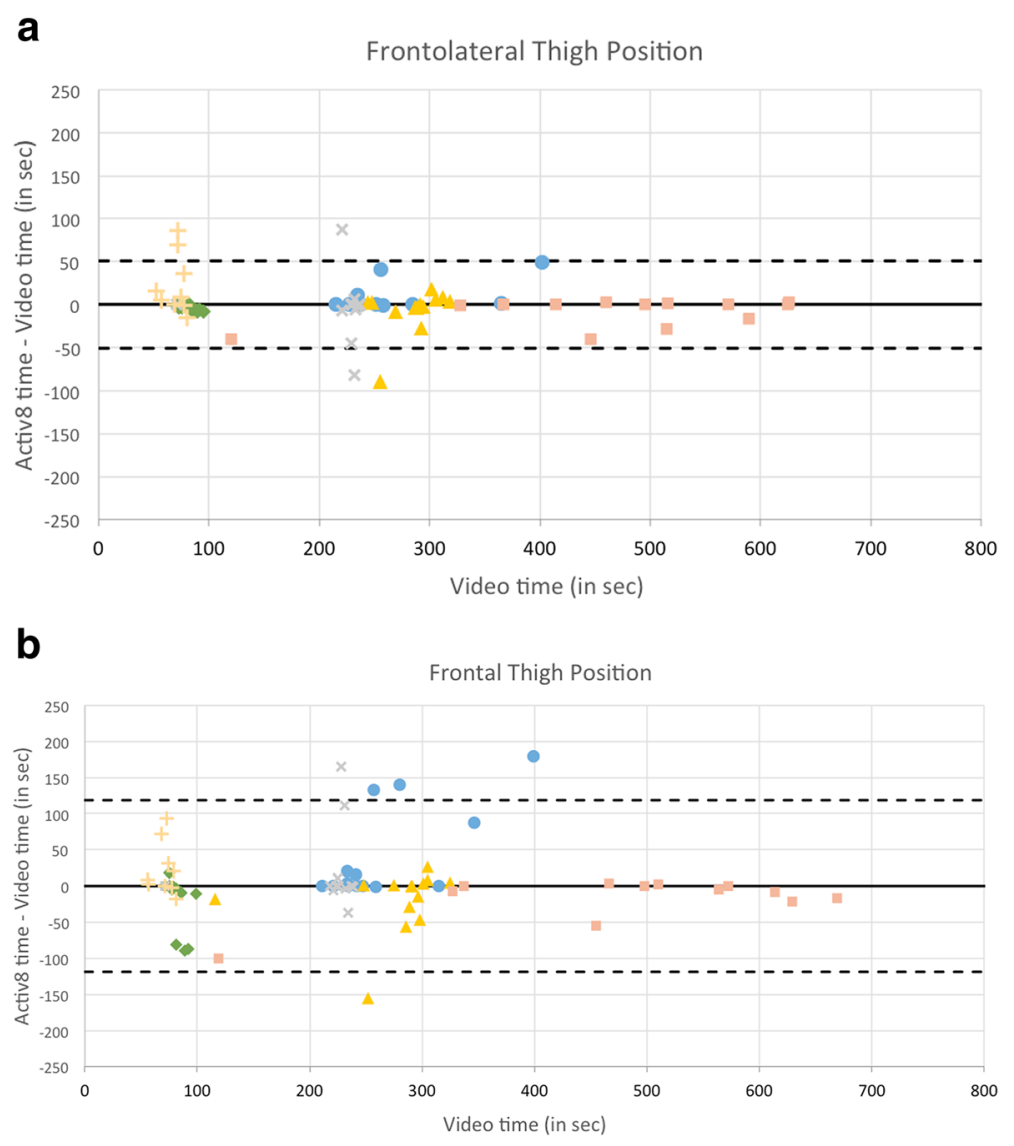

C

Pant Pocket Position



Fig. 2 a. Frontolateral thigh position: Bland-Altman plot (Krouwer's method) between video observation and Activ8 AM. b. Frontal thigh position: Bland-Altman plot (Krouwer's method) between video observation and Activ8 AM. c. Pant pocket position: Bland-Altman plot (Krouwer's method) between video observation and Activ8 AM. a, b, c legend: See attached .png file 
Table 5 Meng's Analysis: Comparison of Spearman Rho correlation coefficients between Activ8 positions and video observation

\begin{tabular}{|c|c|c|c|c|c|c|}
\hline & Sitting & Basic Standing & Basic Walking & Upright Activity $^{a}$ & Bicycling & Running \\
\hline$\overline{r_{1}}$ & 0.98 & 0.93 & 0.94 & 0.99 & 0.49 & 0.73 \\
\hline$r_{2}$ & 0.86 & -0.04 & 0.59 & 0.72 & 0.35 & 0.77 \\
\hline Z-score & 2.96 & 3.60 & 2.88 & 4.82 & 0.42 & 1.36 \\
\hline$p$-value & $.003^{*}$ & $<.001^{*}$ & $.004^{*}$ & $<.001^{*}$ & .672 & .174 \\
\hline$r_{1}$ & 0.98 & 0.93 & 0.94 & 0.99 & 0.49 & 0.73 \\
\hline$r_{3}$ & 0.48 & 0.14 & 0.37 & 0.79 & 0.39 & 0.74 \\
\hline Z-score & 4.16 & 3.66 & 3.07 & 4.31 & 0.31 & 0.30 \\
\hline$p$-value & $<.001^{*}$ & $<.001^{*}$ & $.002^{*}$ & $<.001^{*}$ & .754 & .763 \\
\hline$r_{2}$ & 0.86 & -0.04 & 0.59 & 0.72 & 0.35 & 0.77 \\
\hline$r_{3}$ & 0.48 & 0.14 & 0.37 & 0.79 & 0.39 & 0.74 \\
\hline Z-score & 2.44 & 0.46 & 0.87 & 1.00 & 0.14 & 1.03 \\
\hline$p$-value & $.015^{*}$ & .642 & .384 & .318 & .886 & .304 \\
\hline
\end{tabular}

$r_{1}$ : correlation coefficient for video/frontolateral thigh Activ8; $r_{2}$ : correlation coefficient for video/frontal thigh Activ8; $r_{3}$ : correlation coefficient for video/pant pocket Activ8; a. Upright Activity: the sum of Standing, Standing with Movement, and Walking categories

${ }^{*} p<.05$

activity classes of sitting, walking, and upright activity but was not acceptable for standing (frontal thigh only), bicycling, and running.

An important characteristic of the Activ8 is the ability to distinguish between a set of body P\&M categories. Most activity monitors assess the amount of PA or merely step counts, and not the type of PA [29-31]. However, systems such as the activPAL, Dynaport, and Vitaport/VitaMove also provide body P\&M information. Compared to these systems the Activ8 is inexpensive, easy-to-use, and can provide direct feedback. A recent systematic review and meta-analysis has reported that AMs that provide users with ongoing feedback have moderately positive effects on levels of PA [36]. This suggests AMs like the Activ8 would be valuable components of PA promoting programs in the field of rehabilitation.

Since spasticity may lead to distorted body P\&Ms that are different from other populations, we felt that a population-specific validation study in adults with spastic CP had to be performed. Comparison of our results with the results from other validity studies is difficult because of differences in protocol and data analysis; however, our results indicate comparable validity to other AMs that provide body $\mathrm{P} \& \mathrm{M}$ information in individuals with CP [25, 27, 37]. For example, like the activPAL [27, 37], the Activ8 demonstrated strong agreement for sitting, standing, and walking time compared to observation. However, the Activ8 distinguishes the additional body P\&M categories of bicycling and running.

The Activ8, as a objective measure of physical behaviour in terms of body P\&Ms, has been validated before in other populations including healthy adults (unpublished observations) and people after stroke [23]. As described in stroke [23], in adults with CP the Activ8 demonstrated appropriate detection of sitting, standing, walking, and cycling during basic activities and appropriate detection of upright activity during more complex daily activities. Additionally, this study further highlights the importance of AM placement on body $P \& M$ detection, which was a principal point of discussion in former Activ8 research as well [23].

Our results highlight important considerations with video observation and use of the Activ8 AM. Firstly, the distinction between standing and walking in the video was not always easy to make. In this study, movements that fell between standing and walking were given the classification 'standing with movement' and ranged from minor movements such as when standing restlessly to brief lunges such as when trying to reach a mop underneath a chair. 'Standing with movement' time was often detected as walking by the Activ8 and perhaps appropriately so. The majority of these ambiguous movements were fairly vigorous in nature and may require similar energy expenditure as walking. In this study, we chose to combine standing, walking, and 'standing with movement' activity categories into a pooled 'upright activity' category. Researchers and clinicians have suggested that it is important to promote breaks from SB, in addition to promoting PA, particularly for individuals with $\mathrm{CP}$ [8]. Any break from SB would be captured in the pooled 'upright activity' category during complex PA. Therefore, this pooled category may be appropriate when assessing free-living PA in adults with $\mathrm{CP}$ and would circumvent issues related to 'standing with movement' time. 
In the current Activ8 classification algorithm, all movements surpassing a certain acceleration threshold will be categorized as running, regardless of the unit's angular position, resulting in an overestimation of running time. This was seen in two participants. When asked to bicycle faster than normal, these two participants cycled at notably high intensities, leading to large vector magnitude accelerations. As a result, the correlation between Activ8 AM and video was low to moderate for the detection of bicycling ( $r=$ $0.49)$ and running $(r=0.73)$ despite the average relative time difference being well below $10 \%$ for bicycling (3.19\%). Bicycling is an important activity, particularly in the Netherlands, and therefore inappropriate detection of vigorous bicycling as running time is a shortcoming of the Activ8. Based on the current classification algorithm, 'running' is not an appropriate title for this activity class and should be changed to 'vigorous movement' or 'high intensity activity' to better depict the detected movement.

Lastly, the positioning of the monitor is another important consideration, particularly for patients with hypertonicity, secondary musculoskeletal deformities, and deviated gait patterns. Muscle spasticity and contractures across joints may lead to a crouched stance/ gait pattern [38, 39]. In this study, four participants with prominent crouched stances/gait patterns had standing frequently misclassified as sitting and walking misclassified as bicycling in the frontal thigh Activ8 location. Alternatively, positioning of the Activ8 AM on the frontolateral thigh resulted in more accurate detection of body P\&Ms. At this frontolateral thigh location, gravitational/accelerative forces detected by the z-axis accelerometer are not as large as those detected by the frontal thigh monitor, leading to proper categorization of crouched stance and gait. One of the disadvantages with the frontolateral thigh position is that it is more difficult to standardize. If patients were asked to place the monitor on their own thigh, instructions to attach the monitor to the frontal thigh, along the midline of the thigh, are much simpler than instructions for the frontolateral thigh position.

\section{Study limitations}

This study was only able to evaluate the validity of the Activ8 in a small sample of ambulatory adults with CP. However, when contrasting median and average relative time differences for the detection of each body P\&M and inspecting the Bland-Altman plots, it is evident that gross misclassification of activity time by AM measures is only seen in one or two outliers. With a larger sample of adults, one could expect the average relative time difference to fall closer to the median, further supporting the validity of the Activ8 AM.

Another limitation to this study is use of only one rater to code video recordings. Previous validation studies using video (or direct) observation as criterion measures have used two raters to categorize observed activity for each 1-s frame, later comparing the scores of the raters and determining the inter-rater reliability $[25,27,37]$. In this study, potential bias when rating video recordings was minimized by using rigid definitions of body P\&M categories as well as highlighting ambiguous video and discussing those video data with a researcher experienced in video coding.

The results of this study are limited to ambulatory adults with CP only, as validity will need to be established among other groups of ambulatory adults with impaired gait patterns as well as among non-ambulatory individuals. Future research to establish the validity of a Wheelchair Activ8 configuration would be invaluable to allow to researchers to assess the physical behaviour of a broad range of functional abilities, including nonambulatory adults with neurological conditions such as $\mathrm{CP}$, spinal cord injury, or stroke.

\section{Conclusion}

The Activ8 shows promise as an objective measurement tool to assess physical behaviour in ambulatory adults with spastic CP. This device has shown adequate criterion validity in the detection of sitting, standing, walking, bicycling, and upright activity body $P \& M$ categories in a simulated free-living environment. Proper positioning of the AM is paramount to accurate body P\&M detection, with attachment of the $\mathrm{AM}$ to the frontolateral thigh position resulting in better body P\&M detection than frontal and pant pocket positions. The use of the Activ8 AM in larger clinical studies, inpatient, or outpatient settings should be explored further.

\section{Abbreviations}

AM: Activity monitor; CP: Cerebral palsy; FIFO: First in, first out; GMFCS: Gross motor function classification system; HPA: Habitual physical activity; P\&Ms: Postures and movements; PA: Physical activity; SB: Sedentary behaviour

\section{Acknowledgements}

The authors thank the staff at Erasmus MC and Rijndam Rehabilitation for their providing equipment and space. Thank you to Malou Fanchamps for her assistance with Activ8 data preparation and cleaning. A special thank you is owing to all participating adults and their parents, significant others, or caregivers for their engagement during this study.

\section{Suppliers}

a. Activ8, trademark of Remedy Distribution Ltd. (original equipment manufacturer is $2 \mathrm{M}$ Engineering Ltd.), John F Kennedylaan 3, 5555XC, Valkenswaard, The Netherlands 


\section{Authors' contributions}

EC designed the project, collected, analysed and interpreted the data, and spearheaded writing the manuscript. $\mathrm{HH}$ helped with project design, data analysis, and data interpretation. WS helped with participant recruitment and manuscript preparation. NS played a major role in participant recruitment and data collection. AT and BT both contributed to project design and data interpretation. JWG was involved in project design, data interpretation, and manuscript preparation. RB and JB were both involved in conception and design of this work, as well as interpretation of the data and contributed to writing the manuscript. All authors read and approved the final manuscript.

\section{Funding}

This research did not receive any specific grant from funding agencies in the public, commercial, or not-for-profit sectors.

\section{Availability of data and materials}

The datasets used and/or analysed during the current study are available from the corresponding author on reasonable request.

\section{Ethics approval and consent to participate}

The study was approved by the Medical Ethics Committee of Erasmus MC University Medical Centre, Rotterdam, NL. All participants provided written informed consent.

\section{Consent for publication}

Not applicable.

\section{Competing interests}

The results of this study were presented at the European Academy for Childhood Disability in Amsterdam, May 2017. The authors declare they have no competing interests.

\section{Author details}

'School of Rehabilitation Sciences, McMaster University, 1400 Main St. W, Hamilton, Ontario L8S 1C7, Canada. ${ }^{2}$ CanChild Centre for Childhood Disability Research, McMaster University, 1400 Main St. W, Hamilton, Ontario L8S 1C7, Canada. ${ }^{3}$ Department of Rehabilitation Medicine, Erasmus MC University Medical Centre, P.O. Box 2040, 3000 Rotterdam, CA, Netherlands. ${ }^{4}$ Rijndam Rehabilitation, Westersingel 300, 3015 Rotterdam, L, Netherlands, ${ }^{5}$ Child Health \& Exercise Medicine Program, Department of Pediatrics, McMaster University, 1280 Main St. W, Hamilton, Ontario L8S 4L8, Canada.

\section{Received: 18 April 2019 Accepted: 12 September 2019}

\section{Published online: 29 October 2019}

\section{References}

1. Penedo FJ, Dahn JR. Exercise and well-being: a review of mental and physical health benefits associated with physical activity. Curr Opin Psychiatry. 2005;18(2):189-93.

2. Haskell W, Lee I, Pate R, Powell K, Blair S, Franklin B. Physical actvity and public health: updated recommendation for adults from the American College of Sports Medicine and the American Heart Association. Med Sci Sports Exerc. 2007;39(8):1423-34.

3. Thorpe D. The role of fitness in health and disease: status of adults with cerebral palsy. Dev Med Child Neurol. 2009;51:52-8.

4. Manns PJ, Dunstan DW, Owen N, Healy GN. Addressing the nonexercise part of the activity continuum: a more realistic and achievable approach to activity programming for adults with mobility disability? Phys Ther. 2012; 92(4):614-25.

5. Warburton D, CHarlesworth S, Ivey A, Nettlefold L, Bredin S. A systematic review of the evidence for Canada's physical activity guidelines for adults. Int J Behav Nutr Phys Act. 2010;7:39.

6. Thorp A, Owen N, Neuhaus M, Dunstan D. Sedentary behaviours and subsequent health outcomes in adults: a systematic review of longitudinal studies, 1996-2011. Am J Prev Med. 2011;41(2):207-15.

7. Pedersen BK, Saltin B. Exercise as medicine - evidence for prescribing exercise as therapy in 26 different chronic diseases. Scand J Med Sci Sports. 2015;25:1-72.

8. Verschuren O, Peterson MD, Balemans AC, Hurvitz EA. Exercise and physical activity recommendations for people with cerebral palsy. Dev Med Child Neurol. 2016;58:798-808.
9. Thornton JS, Frémont P, Khan K, Poirier P, Fowles J, Wells GD, et al. Physical activity prescription: a critical opportunity to address a modifiable risk factor for the prevention and management of chronic disease: a position statement by the Canadian academy of sport and exercise medicine. $\mathrm{Br} J$ Sports Med. 2016;50:1109-14.

10. Claridge EA, McPhee PG, Timmons BW, Ginis KAM, MacDonald MJ, Gorter JW. Quantification of physical activity and sedentary time in adults with cerebral palsy. Med Sci Sports Exerc. 2015;47(8):1719-26.

11. van der Slot W, Roebroeck M, Landkroon A, Terburg M, van den BergEmons RJG, Stam H. Everyday physical activity and community participation of adults with hemiplegic cerebral palsy. Disabil Rehabil. 2007;29(3):179-89.

12. Nieuwenhuijsen C, van der Slot W, Beelen A, Arendzen J, Roebroeck M, Stam $\mathrm{H}$, et al. Inactive lifestyle in adults with bilateral spastic cerebral palsy. J Rehabil Med. 2009;41(5):375-81.

13. Hombergen SP, Huisstede BM, Streur MF, Stam HJ, Slaman J, Bussmann JB, et al. Impact of cerebral palsy on health-related physical fitness in adults: systematic review. Arch Phys Med Rehabil. 2012;93(5):871-81.

14. Ryan JM, Crowley VE, Hensey O, Broderick JM, McGahey A, Gormley J. Habitual physical activity and cardiometabolic risk factors in adults with cerebral palsy. Res Dev Disabil. 2014;35(9):1995-2002.

15. Morgan P, McGinley J. Gait function and decline in adults with cerebral palsy: a systematic review. Disabil Rehabil. 2014;36(1):1-9.

16. Opheim A, Jahnsen R, Olsson E, Stanghelle JK. Walking function, pain, and fatigue in adults with cerebral palsy: a 7-year follow-up study. Dev Med Child Neurol. 2009;51(5):381-8.

17. Warburton DER, Nicol CW, Bredin SSD. Health benefits of physical activity: the evidence. Can Med Assoc J. 2006;174(6):801-9.

18. Sallis R, Franklin B, Joy L, Ross R, Sabgir D, Stone J. Strategies for promoting physical activity in clinical practice. Prog Cardiovasc Dis. 2015;57(4):375-86.

19. Prince S, Adamo K, Hamel M, Hardt J, Gorber S, Tremblay M. A comparison of direct versus self-report measures for assessing physical activity in adults: a systematic review. Int J Behav Nutr Phys Act. 2008;5:56

20. Slaman J, Roebroeck ME, van Meeteren J, van der Slot WM, ReindersMesselink HA, Lindeman E, et al. Learn 2 Move 16-24: effectiveness of an intervention to stimulate physical activity and improve physical fitness of adolescents and young adults with spastic cerebral palsy; a randomized controlled trial. BMC Pediatrics. 2010;10(1):79.

21. Prince S, Saunders T, Gresty K, Reid R. A comparison of the effectiveness of physical activity and sedentary behaviour interventions in reducing sedentary time in adults: a systematic review and meta-analysis of controlled trials. Obes Rev. 2010;15(11):905-19.

22. John D, Freedson P. ActiGraph and Actical physical activity monitors: a peek under the Hood. Med Sci Sports Exerc. 2012;44(1 Suppl 1):S86-9.

23. Fanchamps M, Horemans H, Ribbers G, Stam H, Bussmann J. The accuracy of the detection of body postures and movements using a physical activity monitor in people after a stroke. Sensors. 2018;18(7):2167.

24. Cerebral Palsy (CP). Centers for Disease Control and Prevention. 2015.

25. Nooijen CF, de Groot JF, Stam HJ, van den Berg-Emons RJ, Bussmann HB. Validation of an activity monitor for children who are partly or completely wheelchair-dependent. J Neuroeng Rehabil. 2015;12:11.

26. Postma K, van den Berg-Emons HJG, Bussmann JBJ, Sluis TAR, Bergen MP, Stam HJ. Validity of the detection of wheelchair propulsion as measured with an activity monitor in patients with spinal cord injury. Spinal Cord. 2005;43(9):550-7.

27. Tang KT, Richardson AM, Maxwell D, Spence WD, Stansfield BW. Evaluation of an activity monitor for the objective measurement of free-living physical activity in children with cerebral palsy. Arch Phys Med Rehabil. 2013;94(12):2549-58.

28. Usuba K, Oddson B, Gauthier A, Young NL. Leisure-time physical activity in adults with cerebral palsy. Disabil Health J. 2015;8(4):611-8.

29. O'Neil ME, Fragala-Pinkham M, Lennon N, George A, Forman J, Trost SG. Reliability and validity of objective measures of physical activity in youth with cerebral palsy who are ambulatory. Phys Ther. 2016;96(1):37-45.

30. Tudor-Locke C, Sisson SB, Lee SM, Craig CL, Plotnikoff RC, Bauman A. Evaluation of quality of commercial pedometers. Can J Public Health. 2006; 97(Suppl 1: S10-5):S10-6.

31. TJM K, Dontje ML, Sprenger SR, Krijnen WP, van der Schans CP, de Groot M. Reliability and validity of ten consumer activity trackers. BMC Sports Sci Med Rehabil. 2015;7(1):24.

32. Bland JM, Altman DG. Statistical methods for assessing agreement between two methods of clinical measurement. Lancet. 1986;327(8476):307-10. 
33. Krouwer JS. Why Bland-Altman plots should useX, not $(Y+X) / 2$ when $X$ is a reference method. Stat Med. 2008;27(5):778-80.

34. Landis JR, Koch GG. The measurement of observer agreement for categorical data. Biometrics. 1977;33(1):159-74.

35. Meng $X$, Rosenthal R, Rubin DB. Comparing correlated correlation coefficients. Psychol Bull. 1992;111(1):172-5.

36. Braakhuis $H$, Berger $M$, Bussmann J. Effectiveness of healthcare interventions using objective feedback on physical activity: a systematic review and metaanalysis. J Rehabil Med. 2019;51(3):151-9.

37. O'Donoghue D, Kennedy N. Validity of an activity monitor in young people with cerebral palsy gross motor function classification system level I. Physiol Meas. 2014;35(11):2307-18.

38. Sutherland DH, Davids JR. Common gait abnormalities of the knee in cerebral palsy. Clin Orthop Relat Res. 1993;288(1):139-47.

39. Thibaut A, Chatelle C, Ziegler E, Bruno M-A, Laureys S, Gosseries O. Spasticity after stroke: physiology, assessment and treatment. Brain Inj. 2013;27(10):1093-105.

\section{Publisher's Note}

Springer Nature remains neutral with regard to jurisdictional claims in published maps and institutional affiliations.

Ready to submit your research? Choose BMC and benefit from:

- fast, convenient online submission

- thorough peer review by experienced researchers in your field

- rapid publication on acceptance

- support for research data, including large and complex data types

- gold Open Access which fosters wider collaboration and increased citations

- maximum visibility for your research: over $100 \mathrm{M}$ website views per year

At BMC, research is always in progress.

Learn more biomedcentral.com/submissions 